\title{
The effects of diurnal Ramadan fasting on energy expenditure and substrate oxidation in healthy men
}

\author{
Sana'a A. Alsubheen ${ }^{1}$, Mohammad Ismail ${ }^{1}$, Alicia Baker ${ }^{1}$, Jason Blair ${ }^{1}$, Adeboye Adebayo ${ }^{1}$, Liam Kelly ${ }^{2}$, \\ Vikram Chandurkar $^{2}$, Sukhinder Cheema ${ }^{3}$, Denis R. Joanisse ${ }^{4}$ and Fabien A. Basset ${ }^{1 *}$ \\ ${ }^{1}$ School of Human Kinetics and Recreation, Memorial University, St John's, NL, A1C 5S7, Canada \\ ${ }^{2}$ Faculty of Medicine, Memorial University, St John's, NL, A1B 3V6, Canada \\ ${ }^{3}$ Department of Biochemistry, Faculty of Science, Memorial University, St John's, NL, A1B 3X9, Canada \\ ${ }^{4}$ Département de Kinésiologie, Faculté de Médecine, Université Laval, Québec, QC, G1R 7P4, Canada
}

(Submitted 31 March 2017 - Final revision received 18 October 2017 - Accepted 23 October 2017 - First published online 4 December 2017)

\section{Abstract}

The study aimed to examine the effects of diurnal Ramadan fasting (RF) on substrate oxidation, energy production, blood lipids and glucose as well as body composition. Nine healthy Muslim men (fasting (FAST) group) and eight healthy non-practicing men (control (CNT) group) were assessed pre- and post-RF. FAST were additionally assessed at days 10, 20 and 30 of RF in the morning and evening. Body composition was determined by hydrodensitometry, substrate oxidation and energy production by indirect calorimetry, blood metabolic profile by biochemical analyses and energy balance by activity tracker recordings and food log analyses. A significant group $\times$ time interaction revealed that chronic RF reduced body mass and adiposity in FAST, without changing lean mass, whereas CNT subjects remained unchanged. In parallel to these findings, a significant main diurnal effect (morning $v$. evening) of RF on substrate oxidation (a shift towards lipid oxidation) and blood metabolic profile (a decrease in glucose and an increase in total cholesterol and TAG levels, respectively) was observed, which did not vary over the course of the Ramadan. In conclusion, although RF induces diurnal metabolic adjustments (morning $v$. evening), no carryover effect was observed throughout RF despite the extended daily fasting period (18.0 (SD 0.3) h) and changes in body composition.

Key words: Indirect calorimetry: Substrate oxidation: Ramadan: Lipid profiles: Insulin: Body composition

Ramadan, the fasting month of Muslims, occurs in the 9th month of the lunar calendar. During this month, Muslims all over the world abstain from eating, drinking and smoking from local sunrise till local sunset. As it is based on the lunar calendar, Ramadan begins $11 \mathrm{~d}$ earlier each subsequent year and can thus occur in any of the four seasons over time. Therefore, depending on the season and the geographical location, daytime fasting varies from approximately $11-18 \mathrm{~h}$, being longer in the summer and in the temperate regions ${ }^{(1)}$. During Ramadan, food and liquid are usually consumed in two meals, in the morning before sunrise and in the evening after sunset, shifting the pattern of energy intake from daytime to the hours of darkness ${ }^{(2)}$. These changes in the timing of food intake as well as in the composition of diet can alter energy metabolism, as previously reported ${ }^{(3,4)}$. Further to these changes, diurnal dehydration induces additional metabolic perturbations ${ }^{(5)}$. Studies conducted to examine the effect of Ramadan fasting (RF) on energy metabolism and body composition have not reached consensus. Most studies, including a metaanalysis ${ }^{(6)}$, have reported a decrease in body mass $(\mathrm{BM})^{(3,7,8)}$, but not all ${ }^{(4)}$. Reports on changes in blood metabolic profile are also inconsistent and contingent on the quantity and quality of the diet and on body composition ${ }^{(1)}$.

Despite the above-reported discrepancies between studies, $\mathrm{RF}$ remains an interesting alternative model to investigate the beneficial effect of intermittent fasting (IF) and its potential to mitigate chronic diseases in the general population, as suggested by Harder-Lauridsen et al. ${ }^{(9)}$. In this regard, the period of Ramadan in 2015 provided a unique opportunity to enquire into the effect of a long period of diurnal IF on energy metabolism and body composition. Notably, Muslims of Newfoundland were set to fast during the summer season in this temperate region, resulting in fasting periods of approximately $18 \mathrm{~h} / \mathrm{d}$.

Therefore, the objectives of the study were to examine the effect of long diurnal IF throughout Ramadan on energy metabolism, blood metabolic profile and body composition.

Abbreviations: $\mathrm{BM}$, body mass; $\mathrm{CHO}$, carbohydrate; $\mathrm{CHO}_{\mathrm{ox}}$, carbohydrate oxidation; $\mathrm{CNT}$, control; EE, energy expenditure; $\mathrm{EP}$, energy production; $\mathrm{FAST}$, fasting; FAT, fat; $\mathrm{FAT}_{\mathrm{ox}}$, lipid oxidation; HR, heart rate; IF, intermittent fasting; MR, metabolic rate; PRO, protein; RF, Ramadan fasting; R1, 10th day of RF; R2, 20th day of RF; R3, 30th day of RF; VS, vital signs.

* Corresponding author: F. A. Basset, fax +1 709864 3979, email fbasset@mun.ca 
It was hypothesised that this extended IF during Ramadan would alter the diurnal contribution of substrates to energy production (EP), and that this would be magnified by the cumulative metabolic stress over time, contributing to an expected moderate weight loss. It was expected that the contribution of lipid to EP would increase during the day as well as over the course of the month. Along with an altered substrate contribution to EP, the blood serum levels of total cholesterol, TAG, glucose and insulin were predicted to reflect the change in mobilisation, transport and oxidation of substrates

\section{Methods \\ Participants}

The study was approved by the Human Investigation committee of the Health Research Ethics Authority of Newfoundland and Labrador; Memorial University of St John's, Newfoundland, Canada (Reference no.: 15.105) and was carried out in accordance with the declaration of Helsinki. In all, seventeen men of various ethnicities partook in the study. Nine healthy adult men of strict Muslim faith - who stringently followed the five pillars of Islam as confirmed by the local Islamic Centre - formed the Ramadan fasting (FAST) group, and eight healthy adult men who did not fast during Ramadan formed the control (CNT) group. Participants provided written informed consent before completing the Physical Activity Readiness Questionnaire (PAR-Q) to screen for any medical conditions including hypertension, cardiorespiratory disease, diabetes, musculoskeletal injuries or a family history of these conditions. Individuals were included in the study if they passed the PAR-Q, had a BMI $<35$ $\mathrm{kg} / \mathrm{m}^{2}$, and had not participated in a structured physical activity programme during the previous 12 months. Screened participants attended an orientation session $20 \mathrm{~d}$ before the onset of $\mathrm{RF}$ in which they were given information about the experimental design, in addition to undertaking anthropometric measurements (height and BM). Participants were instructed to manually record their total food intake and to wear a physical activity tracker to monitor their physical activity level. Anthropometric characteristics of the participants are reported in Table 1.

\section{Study design}

Anthropometrics, vital signs (VS), blood samples and metabolic rate (MR) were collected between 07.00 and 09.00 hours $10 \mathrm{~d}$ pre- and $10 \mathrm{~d}$ post-RF for all participants, and on the 10th (R1), 20th (R2) and 30th (R3) days of Ramadan in the morning (between 07.00 and 09.00 hours) and the evening (between 18.00 and 20.00 hours) for the FAST group. The study timeline can be seen in Fig. 1.

\section{Anthropometry}

Body density was assessed by hydrostatic weighing as previously described $^{(10)}$. Before hydrostatic weighing, height and BM were measured $( \pm 0 \cdot 1 \mathrm{~cm}, \pm 0 \cdot 1 \mathrm{~kg})$. Lung volume and capacity were determined using a spirometer (Micro Medical Inc.). Thereafter, the subjects, immersed in water, were instructed to exhale completely to the point of residual lung volume, at which point a load cell interfaced with a computer

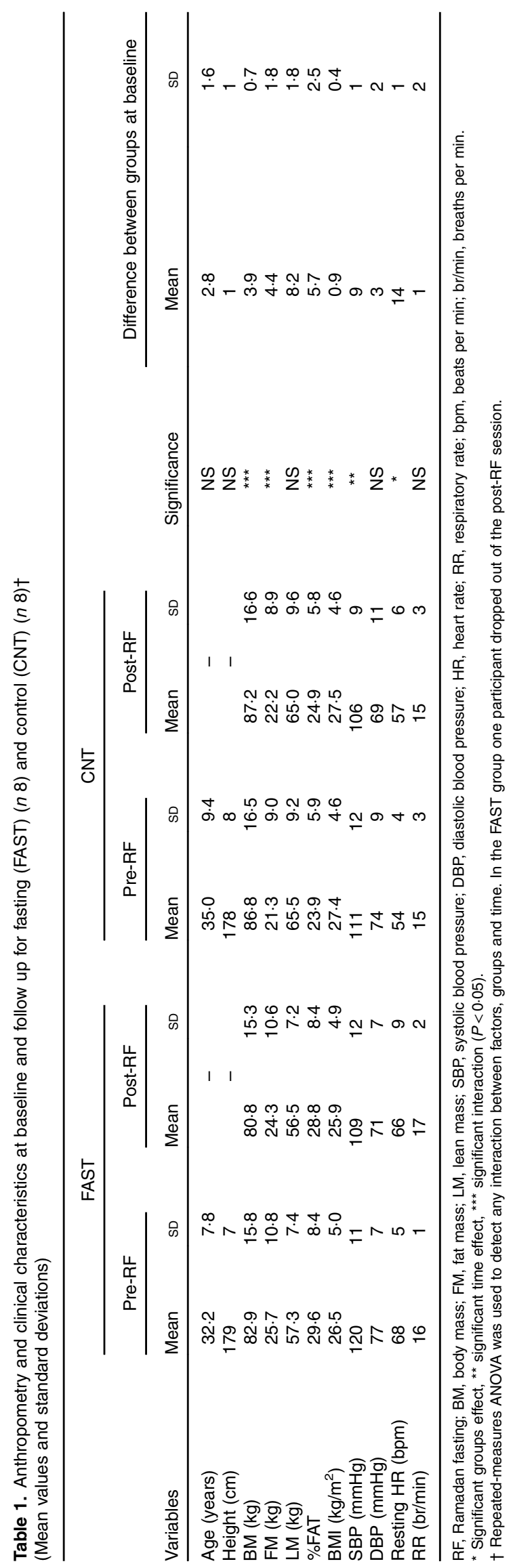




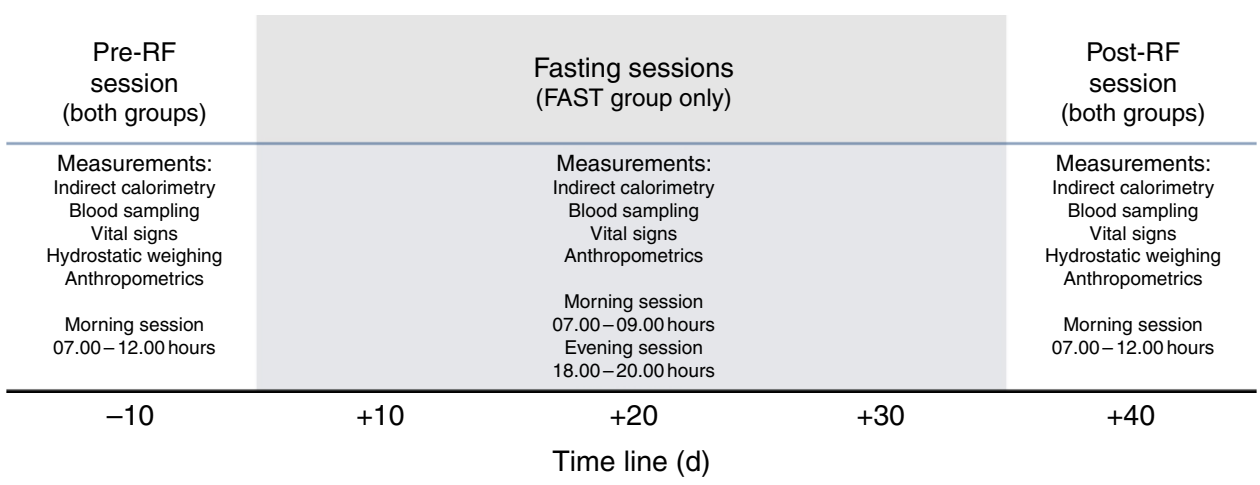

Fig. 1. Experimental design. Time line of the study comprised baseline measurements recorded $10 \mathrm{~d}$ prior to Ramadan fasting (RF), at days 10,20 and 30 during morning and evening sessions within Ramadan, and $10 \mathrm{~d}$ after RF. The anthropometric characteristics, health status and metabolic profile of all participants were determined during the pre- and post-RF sessions. These parameters were also recorded during the Ramadan period on the fasting (FAST) group only, except for body composition. Throughout the study, food intake and physical activity logs were recorded for the FAST and control groups.

was used to obtain the underwater measurement of BM. Measurements were taken until three lung forced vital capacities reached within $0.15 \mathrm{ml}$ the control residual lung volume. The three highest values were, then, averaged ${ }^{(10)}$

\section{Vital signs}

At the beginning of each session, after obtaining BM, VS were collected. Body temperature was measured with an ear thermometer (Braun; Kaz Europe Sàrl) to control for any deviation from normal values, blood pressure (systolic blood pressure (SBP); diastolic blood pressure (DBP)) was recorded with a sphygmomanometer, and heart rate (HR) and respiratory rate (RR) were determined by count over $1 \mathrm{~min}$ timed on a stop watch.

\section{Biochemistry}

A blood sample of approximately $5 \mathrm{ml}$ was drawn from the antecubital vein and collected into Serum and EDTA BD vacutainer tubes for serum and plasma, respectively. These samples were collected under standardised conditions in a supine position after an overnight fast $10 \mathrm{~d}$ pre-RF and $10 \mathrm{~d}$ post-RF, and on R1, R2 and R3 of RF in the morning (postprandial, between 07.00 and 09.00 hours), and in the evening (day fasted, between 18.00 and 20.00 hours) for the FAST group. Unfortunately, no blood parameters were analysed for CNT during the study due to budgetary constraints.

After collecting blood, serum samples were allowed to coagulate. Next, serum and plasma tubes were then centrifuged at $2500 \mathrm{~g}$ at $4^{\circ} \mathrm{C}$ for $15 \mathrm{~min}$. Plasma and serum were separated and stored in cryo-tubes at $-20^{\circ} \mathrm{C}$ until further analysis. Blood parameters were determined through colorimetric method from commercially available assay kits; glucose (Cayman Chemical Company), TAG (Cayman Chemical Company) and total cholesterol (Cell Biolabs, Inc.) and using BioTek PowerWave XS Microplate Spectrophotometer. Insulin was determined by an ELISA (Life Technologies Corporation). Homeostatic model assessment of insulin resistance (HOMA-IR) was calculated using HOMA calculator software available from www.OCDEM. ox.ac.uk and developed by Levy et al. ${ }^{(11)}$. All assays were performed according to manufacturer instructions.

\section{Energy balance}

Throughout the study, participants were required to manually record their total food intake over $3 \mathrm{~d}$ before each session. Food logs were then entered into a web-based programme, TotalCoaching (www.totalcoaching.com) that follows the Canadian nutrient guidelines (www.canada.ca/en/healthcanada/services/canada-food-guides.html), and daily energy intake (DEI; kJ/d (kcal/d)), carbohydrate (CHO; g/d), fat (FAT; $\mathrm{g} / \mathrm{d}$ ) and protein (PRO; g/d) values were determined. Foods consumed were selected from a pre-existing list of foods with complete nutritional information derived from the Canadian nutrient file (https://food-nutrition.canada.ca/cnf-fce/indexeng.jsp). All food logs were reviewed by a trained nutritionist.

Daily physical activity was monitored through a physical activity tracker (Vivofit; Garmin International Inc.), which approximates exercise energy expenditure (EE), the number of steps and distance walked ${ }^{(12)}$. Scores extracted from the physical activity tracker website (www.garminconnect.com) for the purpose of estimating exercise EE that corresponds to any physical activity over resting metabolism ${ }^{(12)}$, step count and distance covered were averaged.

\section{Metabolic rate}

MR was measured through indirect calorimetry. Participants were required for all sessions to rest supine while MR was recorded under a canopy in a thermo-neutral environment $\left(22-24^{\circ} \mathrm{C}\right)$ with dimmed lights. BMR was measured $10 \mathrm{~d}$ pre-RF and $10 \mathrm{~d}$ post-RF for both the FAST and CNT groups. Measurements were taken in the morning between 07.00 and 09.00 hours, for a duration of $45 \mathrm{~min}$ and in an overnight (12 h) fasted state except for ad libitum water. Participants were also instructed to avoid physical activity for $12 \mathrm{~h}$ before coming to the laboratory. To assess the potential effect of energy deficit and the altered substrate partitioning induced by RF, the MR of the FAST group was also recorded at two different time points at days 10, 20, 30 of RF, in the morning (post-prandial, between 07.00 and 09.00 hours), and in the evening (fasted, between 18.00 and 20.00 hours). It is worth noting that only the measurements of pre- and post-RF sessions followed the BMR guidelines as mentioned above. No restriction on food intake, 
physical activity, and caffeine consumption were imposed on FAST and CNT throughout the study.

\section{Indirect calorimetry}

An indirect calorimetry system (Sable Systems International) was used to measure the volume of $\mathrm{O}_{2}$ uptake $\left(\mathrm{VO}_{2}\right)$ and $\mathrm{CO}_{2}$ production $\left(\mathrm{V} \mathrm{CO}_{2}\right)$ simultaneously with a hood canopy. The system was set to record the fractional amount of $\mathrm{O}_{2}$ and $\mathrm{CO}_{2}$, mixing chamber temperature, water vapour pressure, barometric pressure, subsample flow rate and mass flow rate in a negative pressure design. The mass flow generator and controller (FK-500) was set at a rate of 75 litres/min during MR determination. A subsample of that flow (sub-sampler, SS4) was then pulled at $150 \mathrm{ml} / \mathrm{min}$ through a water vapour analyzer (RH-300), a dual IR carbon dioxide analyzer, and a paramagnetic oxygen analyzer (CA-10 Carbon Dioxide and PA-10 Oxygen Analyzers). Fractions of gases in the room were recorded before and after each measurement for baseline references.

Before testing, the $\mathrm{O}_{2}$ and $\mathrm{CO}_{2}$ analyzers were calibrated with room air and reference gases $\left(100 \% \mathrm{~N}_{2}\right.$ and $\left.1 \% \mathrm{CO}_{2}\right)$. In addition, propane gas calibration was performed to ensure accuracy of the reading at a low MR. Water vapour pressure was zeroed after drying samples gases by passing through a column of magnesium perchlorate and the sub-sampler pump was calibrated using a flow meter (Gilmont Rotameter; SigmaAldrich). Gas volumes included in metabolic calculations are expressed at standard conditions of temperature, pressure, and dry from water (STPD).

\section{Calculations}

The respiratory data were truncated by $10 \mathrm{~min}(5 \mathrm{~min}$ at each end) in order to nullify any MR fluctuation due to familiarisation with the ventilated hood and the expected termination of data collection. Respirometry data $\left(\dot{\mathrm{VO}}_{2}\right.$ and $\left.\dot{\mathrm{V}} \mathrm{CO}_{2}\right)$ were then integrated, normalised over time, and corrected for protein oxidation $\left(\mathrm{PRO}_{\mathrm{Ox}}\right)$ at a constant oxidation rate of $0.06 \mathrm{~g} / \mathrm{min}$, and were finally included in the calculation of substrate oxidation $\left(\mathrm{CHO}_{\mathrm{ox}}\right.$ and lipid $\left.\left(\mathrm{FAT}_{\mathrm{Ox}}\right)\right)$. The non-PRO adjusted volumes were obtained by subtracting volume of $\mathrm{O}_{2}$ and $\mathrm{CO}_{2}$ pertaining to $\mathrm{PRO}_{\mathrm{ox}}$ from the total volume of gases, and the substrate oxidation values were used to calculate EP expressed in $\mathrm{kJ} / \mathrm{min}$ ( $\mathrm{kcal} / \mathrm{min}$ ), according to the following formulas ${ }^{(13)}$ :

$$
\begin{aligned}
& \mathrm{CHO}_{\mathrm{OX}}=4.57 \mathrm{VCO}_{2}-3 \cdot 23 \mathrm{VO}_{2}-2 \cdot 60 \mathrm{~N} . \\
& \mathrm{FAT}_{\mathrm{OX}}=1.69 \mathrm{VO}_{2}-1.69 \mathrm{VCO}_{2}-2 \cdot 03 \mathrm{~N} . \\
& \mathrm{EP}=3.74 \mathrm{CHO}_{\mathrm{OX}}+9.46 \mathrm{FAT}_{\mathrm{OX}}+4.32 \mathrm{PRO}_{\mathrm{OX}} .
\end{aligned}
$$

\section{Statistical analyses}

A pilot experiment (three observations) showed that mean RER among participants in post-prandial condition was 0.88 with SD of 0.03 and 0.81 with a sD of 0.03 after $12 \mathrm{~h}$ fasting. We, then, ran a statistical power analysis based on these outcomes ( $G$ *Power, version 3.1) with a total of eight subjects per group (grand total of sixteen) we obtained an effect size of 1.32 for a critical $F_{1,7}$ of 5.59 at a $P=0.05$ and power $(1-\beta$ err prob) of $0 \cdot 92$. Statistical analyses were performed using SPSS, version 23 (SPSS Inc.). All values are reported as means and standard deviations, unless otherwise specified, and an $\alpha$ level $(P)$ of 0.05 was used to indicate statistical significance. First, descriptive statistics were used to explore the data set for homogeneity, sphericity, heteroscedasticity and normality assumptions. Second, statistical inferences between groups over time were assessed for body composition, VS, substrate oxidation and EP through a two-way (two groups (FAST $v$. CNT) $\times 2$ periods (pre$\mathrm{RF}$ and post-RF)) ANOVA with repeated measures. Third, a twoway ( 2 groups $($ FAST $v$. CNT) $\times 5$ periods (pre-RF, R1, R2, R3, post-RF)) ANOVA with repeated measures was run on physical activity scores (kJ (kcal), step count and distance), and food log outcomes (kJ (kcal), FAT, PRO and CHO contents). Finally, a two-way (2 time (morning/evening) $\times 3$ periods (R1, R2, R3)) ANOVA with repeated measures was computed on substrate oxidation, EP, blood parameters, body composition and VS to detect the changes induced by the daytime fasting through $\mathrm{RF}$, for FAST only. Significant interactions were followed by pairwise comparisons using a Bonferroni correction.

\section{Results}

For sake of simplicity the main four factors from statistical analyses were denoted group (FAST $v$. CNT), pre- to post-RF (pre- and post-measurements)], daytime fasting (for changes from morning to evening), time (for changes within Ramadan).

\section{Comparisons between fasting and control groups}

Anthropometric data in the FAST and CNT groups are given in Table 1. Note that one participant in the FAST group dropped out of the post-RF session, reducing the sample size to eight. Significant interactions between groups and pre- to post-RF were observed on BM $\left(F_{1,7}=8 \cdot 241 ; P=0.024\right), \quad F M \quad\left(F_{1,7}=11 \cdot 419\right.$; $P=0.012)$, BMI $\left(F_{1,7}=7 \cdot 297 ; P=0.031\right)$ and \%FAT $\left(F_{1,7}=9.084\right.$; $P=0.020)$. The pairwise comparisons showed that, pre- to post$\mathrm{RF}$, IF induced a decrease in BM, FM, BMI and \%FAT for the FAST group, whereas all of these factors remained stable in CNT. Except for a significant main effect of group on resting $\mathrm{HR}$ $\left(F_{1,7}=15.051 ; P=0.006\right)$, and a significant main effect of pre- to post-RF on SBP $\left(F_{1,7}=6.573 ; P=0.037\right)$, no other significant changes were detected in VS. The significant difference in HR reflects fitness differences between groups - CNT participants were more active (see the 'Energy balance' section). Finally, substrate oxidation (glucose and lipids), RER, and EP remained unchanged pre- to post-RF in both groups. These indirect calorimetry outcomes confirmed that metabolic profiles within and between participants at baseline (pre-treatment) were quite similar.

Statistical analysis on blood parameters for the FAST group revealed no significant difference between pre- and post-RF on glucose $($ pre-RF $=4.4(\mathrm{sD} \mathrm{0.6}) \mathrm{mmol} / \mathrm{l} ;$ post-RF $=4.2(\mathrm{sD} \mathrm{0.8}) \mathrm{mmol} / \mathrm{l})$, insulin $(\mathrm{pre}-\mathrm{RF}=86(\mathrm{sD}$ 81) $\mathrm{pmol} / \mathrm{l} ;$ post-RF $=66(\mathrm{sD} 42) \mathrm{pmol} / \mathrm{l})$, total cholesterol (pre-RF $=3.8 \quad(\mathrm{sD} \quad 0.8) \mathrm{mmol} / \mathrm{l} ; \quad$ post-RF $=4 \cdot 0$ $(\mathrm{sD} \quad 0.9) \mathrm{mmol} / \mathrm{l}$ ) and $\mathrm{TAG}$ (pre-RF $=0.8$ (sD 0.6$) \mathrm{mmol} / \mathrm{l}$; post$\mathrm{RF}=1.3(\mathrm{sD} 1 \cdot 1) \mathrm{mmol} / \mathrm{l})$, and consequently HOMA-IR, calculated 
using insulin and glucose levels in fasting state, was not significantly changed following $\mathrm{RF}$ (pre-RF $=1.5$ (sD 1.2) units; post-RF $=1 \cdot 2$ (sD 0.6) units).

As displayed in Table 2, the two-way ANOVA ( 2 groups $\times 5$ periods) with repeated measures on DEI (kJ (kcal), CHO, FAT, PRO intake) revealed no significant difference between FAST and CNT or between periods (pre-, R1, R2, R3, post-RF).

Although the contribution of CHO, FAT and PRO to the DEI did not significantly change throughout the study in either group, it is worth noting that, in FAST, CHO intake increased from pre-RF to R1 by $15 \%$ and then decreased from R1 to post-RF by $29 \%$. FAT intake slightly decreased throughout the study compared with pre-RF values in both groups (FAST $-8 \%$; CNT $-16 \%$ ). For PRO content of food, no variation in CNT, whereas a slight increase $(+9 \%)$ occurred in FAST during RF.

Physical activity tracker used (Vivofit) does not distinguish between physical activity and non-physical activity thermogenesis ${ }^{(12)}$. The Vivofit software merges these parameters in one category called 'active energy content' that corresponds to $\mathrm{EE}$ over resting state. Therefore, for our statistical analyses, EE included all physical activity intensities. Note that one CNT participant was removed from the analysis due to an EE scores over $3 \mathrm{sD}$ from the mean group (EE: $3761 \mathrm{~kJ} / \mathrm{d}(899 \mathrm{kcal} / \mathrm{d}$ ), steps: 20968 steps $/ \mathrm{d}$, distance covered: $18 \mathrm{~km} / \mathrm{d}$ ). In the current study, EE $\left(F_{1,6}=7.758 ; P=0.03\right)$, daily step count $\left(F_{1,6}=8 \cdot 148\right.$; $P=0.03)$ and total distance covered $\left(F_{1,6}=6.636 ; P=0.04\right)$ all showed a significant main effect of group. The CNT actively spent 1197 (sD 1109) kJ/d (286 (sD 265) kcal/d) compared with 757 (sD 506) kJ/d (181 (sD 121) kcal/d) for FAST. Accordingly, daily step count and total distance covered were also higher in CNT compared with FAST. Indeed, CNT cumulated a daily step count of 9685 (SD 7126) and covered a total distance of 7.4 (SD 5.8$) \mathrm{km} / \mathrm{d}$, whereas FAST cumulated a daily step count of 6850 (sD 2079) step and summed a total distance of 5.1 (SD 1.8) km/d.

Comparisons within conditions (post-prandial and fasting), and between days of Ramadan (fasting group only)

A significant main effect of daytime fasting (morning $v$. evening) $\left(F_{2,14}=6.641 ; P=0.009\right)$ as well as a significant main effect of time (R1 to R3) $\left(F_{1,7}=73.124 ; P=0.001\right)$ on BM were observed. BM loss from morning to evening for all time points of Ramadan were similar, and averaged 1.4 (SD 1.4) kg for R1, R2 and R3. The rate of weight loss between R2 and R3 (0.6 (sD 3.4$) \mathrm{kg}$ ) was lower than between R1 and R2 (1.0 (SD $2 \cdot 3) \mathrm{kg}$ ).

For SBP, DBP, RR and HR, no significant main effect of time (R1 to R3) was observed. However, there was a significant main effect of daytime fasting (morning $v$. evening) $\left(F_{1,7}=6 \cdot 236\right.$; $P=0.04)$ on RR showing lower values during evening sessions (15.9 (sD 2.6) breaths per $\mathrm{min}(\mathrm{br} / \mathrm{min})$ ) compared with morning sessions (17.1 (SD 4.8) br/min).

A significant main effect of daytime fasting was observed on $\mathrm{CHO}_{\text {ox }}\left(F_{1,7}=64.640 ; \quad P=0.001\right)$ and $\mathrm{FAT}_{\mathrm{Ox}}\left(F_{1,7}=53.240\right.$; $P=0.001$ ) at all time points during RF (Fig. 2). These changes in substrate oxidation mirrored a significant decrease in RER values $\left(F_{1,7}=84.160 ; P=0.001\right)$ from morning ( 0.88 (sD 0.04$\left.)\right)$ to evening $(0.82$ (sD 0.05$))$. In meantime, EP was not significantly different 


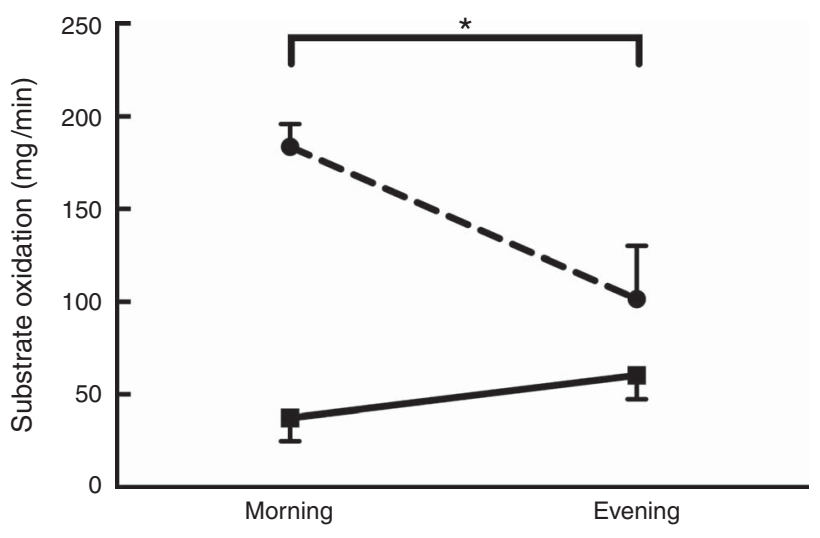

Fig. 2. Substrate oxidation as a function of daytime fasting. Measurements were recorded during Ramadan fasting: ——, lipid oxidation; - - carbohydrate oxidation. Substrate oxidation was measured by indirect calorimetry and calculated using the equations of Simonson \& DeFronzo ${ }^{(13)}$. * Significant time effect $(P<0.05)$

from morning to evening (5.4 (SD 1.7) v. $5 \cdot 0$ (SD 2.1) $\mathrm{kJ} / \mathrm{min}$ $(1.3(\mathrm{SD} 0.4) v \cdot 1 \cdot 2(\mathrm{sD} 0.5) \mathrm{kcal} / \mathrm{min})$, respectively).

In parallel with substrate oxidation outcomes, a significant main effect of daytime fasting (morning $v$. evening) was observed on glucose $\left(F_{1,8}=9 \cdot 181 ; P=0.02-\right.$ morning. $=5.5$ (sD 0.8$) \mathrm{mmol} / \mathrm{l}, \quad$ evening $=4.5 \quad(\mathrm{sD} \quad 0.4) \mathrm{mmol} / \mathrm{l})$, insulin $\left(F_{1,7}=6 \cdot 236 ; P=0.04-\right.$ morning $=170(\mathrm{sD} 126) \mathrm{pmol} / \mathrm{l}$, evening $=75(\mathrm{sD} 93) \mathrm{pmol} / \mathrm{l})$, total cholesterol $\left(F_{1,7}=6 \cdot 236 ; P=0.04\right.$ 9 morning $=4.2(\mathrm{sD} 1.0) \mathrm{mmol} / \mathrm{l}$, evening $=3.7(\mathrm{sD} 0 \cdot 8) \mathrm{mmol} / \mathrm{l})$ and TAG $\left(F_{1,7}=6.236 ; \quad P=0.04 \quad-\quad\right.$ morning $=1.60$ $(\mathrm{sD} 1 \cdot 10) \mathrm{mmol} / \mathrm{l}$, evening $=0.68(\mathrm{sD} 0 \cdot 60) \mathrm{mmol} / \mathrm{l})$.

\section{Discussion}

The objectives of this study were to investigate the effects of relatively long-duration diurnal IF throughout Ramadan on energy metabolism, blood metabolic profile, and body composition. The primary outcomes showed that daytime fasting had an impact on the diurnal contribution of substrates to EP and on blood metabolic profile. In addition, RF induced changes in body composition. However, the metabolic responses to IF did not persist after RF.

\section{The overall effect of Ramadan fasting}

It is worth to mention that all participants remained healthy throughout RF and did not complain of any disorders. BM, fat mass, BMI and \%FAT were all reduced from pre- to post-RF in FAST, whereas no changes were observed in CNT. These body composition adjustments might result from the altered contribution of substrate oxidation, as displayed in Fig. 2. The significant higher $\mathrm{FAT}_{\mathrm{ox}}$ during daytime fasting likely compensated for reduced $\mathrm{CHO}$ availability and to spare liver glycogen. In addition to metabolic changes, a small reduction in food intake of about $1452 \mathrm{~kJ} / \mathrm{d}(347 \mathrm{kcal} / \mathrm{d})$ over the course of Ramadan might have induced a negative energy balance and contributed to these outcomes. These results agree with previous findings that reported significant decreases in BM and \% FAT due to a deficit in energy intake ${ }^{(7)}$. However, in the present study, daytime fasting did not significantly alter lean BM (Refer to Table $1 ; \Delta=0 \cdot 8$ (SD 02)) and, therefore, such a finding attenuates the effect of energy intake deficit. These results are in contrast to those of a recent study on healthy lean men during $\mathrm{RF}$ that reported no changes in BM, despite a small but significant change in $\mathrm{BMI}^{(9)}$.

No significant differences between groups were observed post-RF in EP, substrate oxidation, and RER values, as similarly reported in other studies ${ }^{(4,14,15)}$. In agreement with Fakhrzadeh et $a l .{ }^{(16)}$ no changes in VS were found. In contrast, Ünalacak et $a l .{ }^{(17)}$ and Rahman et $a l .{ }^{(18)}$ reported significant decrease in $\mathrm{BP}$, whereas Husain et al. ${ }^{(14)}$ observed a reduction in HR.

As well, blood parameters (glucose, insulin, total cholesterol and TAG) were not significantly changed post-RF; an expected outcome as RF is not a prolonged and severe continuous fast, but rather a month of changed eating schedule ${ }^{(19)}$. Overall, the findings are similar to those of previous studies ${ }^{(4,15,17,19-21)}$.

Our data are not in complete agreement with previous work. Others $^{(9)}$ reported only a small impact of IF on BMI with no change in body composition and substrate metabolism over $28 \mathrm{~d}$. Note that participants from this previous report were fasting for $14 \mathrm{~h} / \mathrm{d}, 4 \mathrm{~h}$ less than the participants in the current study. Significant decreases in glucose levels ${ }^{(17,19)}$ and in TAG levels ${ }^{(16,17)}$ have also been reported following RF. Although one cannot discount technical factors (equipment validity, accuracy, and reliability), lifestyle and cultural differences such as demographic location, total energy intake, type of foods consumed and amounts and types of physical activity ${ }^{(1,22)}$ could certainly contribute to these outcome discrepancies between studies. Another major confounder is undoubtedly daylight hours during Ramadan depending on geographical location and season: daily fasting times that can vary between as short as $10 \mathrm{~h}$ to over $18 \mathrm{~h} / \mathrm{d}$. Lastly, as our subjects displayed a healthy metabolic state at the onset of Ramadan, no large effects on these variables over the course of the rite were expected.

\section{The effects of daytime fasting on the fasting group}

The effect of daytime fasting on substrate oxidation resulted in an increase of $\mathrm{FAT}_{\mathrm{ox}}$ by $62 \%$ with a concurrent decrease of $\mathrm{CHO}_{\text {ox }}$ by $55 \%$ from morning to evening. Despite this change in substrate partitioning, EP did not oscillate much from morning to evening within RF. This contrasts with the data presented by el Ati et $a l .{ }^{(4)}$ who reported significant decreases in EP during daytime fasting ${ }^{(4)}$. However, as noted by Maughan et al. ${ }^{(23)}$, EP does not considerably vary on short-term fasting and the increase in the rate of $\mathrm{FAT}_{\mathrm{Ox}}$ satisfies energy demands and spares the limited availability of $\mathrm{CHO}$ during the post-prandial phase $^{(23)}$. The metabolic stress induced by RF is sufficient to modulate substrate storage and usage ${ }^{(24)}$. Among the many studies investigating RF, only one study examined the change in substrate oxidation in resting state from morning to evening ${ }^{(4)}$. The participants of that study were all healthy women who had shifted from $\mathrm{CHO}_{\mathrm{ox}}$ to $\mathrm{FAT}_{\mathrm{ox}}$, as we observed in our male participants.

In the current study, the shift in fuel oxidation from morning to evening was mirrored by the changes in blood parameters (glucose, insulin, total cholesterol and TAG). Although blood 
glucose levels significantly declined (18\%), they remained within the normal range. Our data support previous research that has reported reduced blood glucose and an increased rate of $\mathrm{FAT}_{\mathrm{ox}}$ in the fasted state during evening sessions ${ }^{(4)}$, a metabolic adjustment that helps to maintain glucose homoeostasis.

During fasting, $\mathrm{CHO}$ intake decreases and triggers the compensatory response of increasing the rate of TAG mobilisation that elevates blood free fatty acids (FFA) and glycerol levels. FFA supply for direct source of energy and glycerol goes through gluconeogenesis in the liver for the production of glucose $^{(25)}$. In this regard, the decreased levels of both total cholesterol by $12 \%$ and TAG by $58 \%$ in the evening sessions in our fasting subjects provides evidence of increased supply of lipids for EP via $\mathrm{FAT}_{\mathrm{Ox}}$ and gluconeogenesis. In agreement, Fakhrzadeh et $a l^{(16)}$ measured the level of total cholesterol during $\mathrm{RF}$ and concluded that the reduction of total cholesterol levels in blood indicates increased fat oxidation ${ }^{(16)}$. These integrated metabolic responses to fasting are regulated by changes in the hormonal environment including a change in the plasma insulin level ${ }^{(25)}$ as our results showed. The greatly reduced insulin levels (by 56\%) observed during evening sessions can promote lipolysis as reported by el Ati et $a l^{(4)}$. In addition, the significantly lower RER values provide indirect evidence of predominant lipolysis in the evening sessions. In contrast to our results, Sweileh et $a l .{ }^{(7)}$ assessed RER in men by collecting resting $\mathrm{O}_{2}$ uptake for $5 \mathrm{~min}$ and reported non-significant changes to RER values between morning and evening sessions during the 1 st and the 4 th weeks of $\mathrm{RF}^{(7)}$. This non-significant result likely stems from a too short $(5 \mathrm{~min}$ ) data collection period to reach metabolic steady-state. When MR was recorded on a longer period of time, el Ati $e t a l .{ }^{(4)}$ reported a significant decline in RER similar to our results and concluded that fat oxidation was dominant in the evening sessions during RF.

VS including SBP, DBP, RR and HR were monitored during the daytime fasting. Our results showed no significant changes in these parameters. This contrasts with a significant decrease in HR of 2.7 beats per min (bpm) in the morning and $14 \mathrm{bpm}$ in the evening observed by Sweileh et al. ${ }^{(7)}$. Finally, the significant decrease in $\mathrm{BM}$ over the course of the daytime fasting might result in part from dehydration as Muslim fasting requires abstinence from drinking during the day ${ }^{(5)}$.

\section{Energy balance}

The observed decrease in daily food intake over the course of $\mathrm{RF}$, although not statistically significant, appears to be of biological relevance and concurs with previous studies that showed decreased total energy intake ${ }^{(7,14,16,19)}$. In fact, when compared with controls, fasting subjects experienced a decrease of $1092 \mathrm{~kJ} / \mathrm{d}(261 \mathrm{kcal} / \mathrm{d})$ from R1 to R3 and $1452 \mathrm{~kJ} / \mathrm{d}$ $(347 \mathrm{kcal} / \mathrm{d})$ from pre- to post-RF. Similar findings were reported by el Ati et $a l^{(4)}$ and Norouzy et $a l .{ }^{(26)}$. These authors concluded that such findings negate the common belief that Muslims tend to overcompensate in term of food intake during $\mathrm{RF}^{(4,26)}$. Although the present study reports insignificant changes in food composition, a reduction in FAT and an increase in PRO consumption were observed within RF. el Ati et al. ${ }^{(4)}$ showed a relative increase in the FAT and PRO contents, with a corresponding decrease in $\mathrm{CHO}$ as the total food intake was unchanged. Some other studies reported significant decreases in FAT $^{(18)}$ and PRO intake ${ }^{(26)}$, and finally, a decrease in both FAT and PRO intake ${ }^{(7)}$. Certainly, these variations in total food intake and composition might be explained by the difference in food habits from Islamic countries ${ }^{(4)}$. Regional and cultural differences where RF occurs should also be taken into consideration $^{(22)}$

The CNT group was significantly more active during RF in terms of total EE, step count and distance covered per $\mathrm{d}$ as compared with the FAST group, the latter showing no significant change in the amount of physical activity throughout RF (Table 2). Similarly, McNeil et al. ${ }^{(15)}$ reported no significant changes in physical activity measured using accelerometers during RF in healthy men that correlated to a lack of change in $\mathrm{BM}$ and body composition ${ }^{(15)}$. In the present study, no changes in $\mathrm{EP}$ and $\mathrm{EE}$ were revealed throughout $\mathrm{RF}$, thus the reduction in $\mathrm{BM}$ and FM observed post-RF might be attributed to a fuel oxidation shift from $\mathrm{CHO}$ to FAT during daytime fasting and to the slight negative energy balance-increase in the difference between EE and food intake; although the latter was not statistically significant

\section{Methodological considerations}

The current study has some methodological considerations that merit discussion. First, the present findings are limited to a small sample size of healthy men with BMI between 25 and $28\left(\mathrm{~kg} / \mathrm{m}^{2}\right)$ living in Newfoundland, Canada, that limits the generalisation of the outcomes to other populations. This relatively small sample size increases the risk of a Type II error, such that changes to some of the dependent variables of interest (e.g. BM change, substrate shift and blood profile alterations) might have been underestimated owing to intra- and intersubject variations. However, the risk of type I errors, a threat to the correct rejection of a null hypothesis, remains unlikely. Second, the missing analyses of blood parameters pre- and post-Ramadan for the CNT group challenged the statistical comparisons. However, the absence of changes in anthropometric, energy intake and exercise parameters, suggests that CNT blood parameters followed the same trend. Third, the study design was such that no data were collected during Ramadan for the CNT group except food intake and EE, limiting the interpretation of between-group comparisons. Fourth, the body composition was evaluated only pre- and immediately post-RF; thus, it is uncertain whether changes persisted over an extended period after RF. Fifth, similar to other published and ongoing studies, food logs completed by the participants are probably underestimating total food intake; however, this under-estimation occurs throughout the course of the study and should be constant within-subject, limiting its effect on longitudinal analyses. Lastly, a study from our laboratory ${ }^{(12)}$ reported that Vivofit activity trackers underestimate total EE (larger under-estimation for lighter activities), therefore, the actual physical activity our subjects could be underreported; again, this error should be constant throughout the study, limiting its impact on longitudinal analyses. 


\section{Conclusion}

Diurnal RF induces change in substrates contribution to EP from morning to evening that partially support our initial hypothesis; however, these metabolic responses do not change over the course of the month of fasting, nor do they persist after Ramadan. Although the changes in body composition appear to result from a small energy deficit, we cannot discard the impact of IF on the observed metabolic adjustments and its potential to mitigate chronic diseases. However, further investigations should determine the length of daytime fasting and duration of fasting period that bring beneficial responses.

\section{Acknowledgements}

The authors would like to thank Dr Alkanani for the technical support and Mr David Gill, the owner of TotalCoaching.com, for providing access to his platform and technical support. Finally, the authors would like to thank the participants for their time, commitment and patience.

The work was supported by an internal grant from the School of Human Kinetics and Recreation, Memorial University, St. John's, NL, Canada. All persons who meet authorship criteria are listed as authors, and all authors certify that they have participated sufficiently in the work to take public responsibility for the content, including participation in the concept, design, analysis, writing, or revision of the manuscript.

Design and conduct of the study and manuscript writing: F. A. B., S. A. A, S. C., D. R. J.; data collection: F. A. B., S. A. A., M. I., J. B., A. A., L. K., D. R. J.; data analysis: F. A. B., S. A. A., D. R. J., A. B.; data interpretation: F. A. B., S. A. A., D. R. J., V. C. The authors declare that there are no conflicts of interest.

\section{References}

1. Azizi F (2010) Islamic fasting and health. Ann Nutr Metab 56 273-282.

2. Chaouachl A, Leiper JB, Souissi N, et al. (2009) Effects of Ramadan intermittent fasting on sports performance and training: A review. Int J Sports Physiol Perform 4, 419-434.

3. Bouhlel E, Salhi Z, Bouhlel H, et al. (2006) Effect of Ramadan fasting on fuel oxidation during exercise in trained male rugby players. Diabetes Metab 32, 617-624.

4. el Ati J, Beji C \& Danguir J (1995) Increased fat oxidation during Ramadan fasting in healthy women: an adaptative mechanism for body-weight maintenance. Am J Clin Nutr $\mathbf{6 2}$, 302-307.

5. Leiper J \& Molla A (2003) Effects on health of fluid restriction during fasting in Ramadan. Eur J Clin Nutr 57, 30-38.

6. Kul S, Savaş E, Öztürk ZA, et al. (2014) Does Ramadan fasting alter body weight and blood lipids and fasting blood glucose in a healthy population? A meta-analysis. J Relig Health $\mathbf{5 3}$, 929-942.

7. Sweileh N, Schnitzler A, Hunter GR, et al. (1992) Body composition and energy metabolism in resting and exercising
Muslims during Ramadan fast. J Sports Med Phys Fitness 32 , 156-163.

8. Ziaee V, Razaei M, Ahmadinejad Z, et al. (2006) The changes of metabolic profile and weight during Ramadan fasting. Singapore Med J 47, 409-414.

9. Harder-Lauridsen NM, Rosenberg A, Benatti FB, et al. (2017) Ramadan model of intermittent fasting for $28 \mathrm{~d}$ had no major effect on body composition, glucose metabolism, or cognitive functions in healthy lean men. Nutrition 37, 92-103.

10. Behnke AR \& Wilmore JH (1974) Evaluation and Regulation of Body Build and Composition. Englewood, NJ: Prentice Hall.

11. Levy JC, Matthews DR \& Hermans MP (1998) Correct homeostasis model assessment (HOMA) evaluation uses the computer program. Diabetes Care 21, 2191-2192.

12. Alsubheen SA, George AM, Baker A, et al. (2016) Accuracy of the vivofit activity tracker. J Med Eng Technol 40, 298-306.

13. Simonson DC \& DeFronzo RA (1990) Indirect calorimetry: methodological and interpretative problems. Am J Physiol 258, E399-E412.

14. Husain R, Duncan MT \& Ch 'ng DSL (1987) Effects of fasting in Ramadan on tropical Asiatic Moslems. Br J Nutr 58, 41-48.

15. McNeil J, Mamlouk MM, Duval K, et al. (2014) Alterations in metabolic profile occur in normal-weight and obese men during the Ramadan fast despite no changes in anthropometry. J Obes 2014, 9.

16. Fakhrzadeh H, Larijani B, Sanjari M, et al. (2003) Effect of Ramadan fasting on clinical and biochemical parameters in healthy adults. Ann Saudi Med 23, 223-226.

17. Ünalacak M, Kara İH, Baltaci D, et al. (2011) Effects of Ramadan fasting on biochemical and hematological parameters and cytokines in healthy and obese individuals. Metab Syndr Relat Disord 9, 157-161.

18. Rahman M, Rashid M, Basher S, et al. (2004) Improved serum HDL cholesterol profile among Bangladeshi male students during Ramadan fasting. East Mediterr Health J 10, 131-137.

19. Khan A \& Khattak MMAK (2002) Islamic fasting: an effective strategy for prevention and control of obesity. Pak J Nutr 1, 185-187.

20. Fedail SS, Murphy D, Salih SY, et al. (1982) Changes in certain blood constituents during Ramadan. Am J Clin Nutr 36, 350-353.

21. Maislos M, Khamaysi N, Assali A, et al. (1993) Marked increase in plasma high-density-lipoprotein cholesterol after prolonged fasting during Ramadan. Am J Clin Nutr 57, 640-642.

22. Maughan RJ, Bartagi Z, Dvorak J, et al. (2008) Dietary intake and body composition of football players during the holy month of Ramadan. J Sports Sci 26, S29-S38.

23. Maughan RJ, Fallah J \& Coyle EF (2010) The effects of fasting on metabolism and performance. Br J Sports Med 44, 490-494.

24. Azizi F (2002) Research in Islamic fasting and health. Ann Saudi Med 22, 186-191.

25. Brooks GA, Fahey TD \& Baldwin KM (2005) Exercise Physiology: Human Bioenergetics and Its Applications. New York: McGraw-Hill Higher Education.

26. Norouzy A, Salehi M, Philippou E, et al. (2013) Effect of fasting in Ramadan on body composition and nutritional intake: a prospective study. J Hum Nutr Diet 26, 97-104. 